\title{
micromachines
}

ISSN 2072-666X

www.mdpi.com/journal/micromachines

Article

\section{Motility Control of Bacteria-Actuated Biodegradable Polymeric Microstructures by Selective Adhesion Methods ${ }^{\dagger}$}

\author{
Hyung Jung Yoo, Sangmin Lee and Dong-il Dan Cho * \\ Inter-University Semiconductor Research Center, Automation System Research Institute, School of \\ Electrical Engineering and Computer Science, Seoul National University, Seoul 151-744, Korea; \\ E-Mails: yhj@snu.ac.kr (H.J.Y.); sangmlee@snu.ac.kr (S.L.) \\ $\dagger \quad$ This paper is an extended version of our paper published in the 7th Asia-Pacific Conference on \\ Transducers and Micro/Nano Technologies (APCOT 2014), Daegu, Korea, 29 June-2 July 2014. \\ * Author to whom correspondence should be addressed; E-Mail: dicho@snu.ac.kr; \\ Tel.: +82-02-880-6488; Fax: +82-02-875-0867.
}

External Editor: Ulrike Wallrabe

Received: 21 August 2014; in revised form: 14 November 2014 / Accepted: 21 November 2014 / Published: 28 November 2014

\begin{abstract}
Certain bacteria have motility and can be made non-toxic, and using them for drug delivery has been proposed. For example, using bacteria with flagella motion in multiple spin actuators in drug delivery microrobots has been suggested. This paper investigates various adhesion enhancement methods for attaching bacteria on preferred surfaces of cubic polymeric microstructures to achieve the directional control of motion. Serratia marcescens which has an excellent swimming behavior and $50-\mu \mathrm{m}$ sized cubic structures made of biodegradable poly-capro-lactone (PCL) are used. Three treatment methods are investigated and compared to the untreated control case. The first method is retarding bacterial attachments by coating certain surfaces with bovine serum albumin (BSA) which makes those surfaces anti-adherent to bacteria. The second and third methods are roughening the surfaces with X-ray irradiation and plasma respectively to purposely increase bacterial attachments on the roughened surfaces. The measured motilities of bacteria-tethered PCL microactuators are $1.40 \mu \mathrm{m} / \mathrm{s}$ for the BSA coating method, $0.82 \mu \mathrm{m} / \mathrm{s}$ for the X-ray irradiation, and $3.89 \mu \mathrm{m} / \mathrm{s}$ for the plasma treatment method. Therefore, among the methods investigated in the paper the plasma treatment method achieves the highest directionality control of bacteria motility.
\end{abstract}


Keywords: microactuators; microrobot; targeted drug delivery; bacteria; bacteria-tethering; Serratia marcescens; selective bacterial attachment; BSA coating; X-ray irradiation; plasma treatment; biodegradable polymer

\section{Introduction}

Drug delivery systems (DDSs) which deliver drugs to target cells have drawn significant attention recently [1]. To develop effective drug delivery systems, a time-controlled and site-specific drug release method is necessary [2]. By encapsulating drugs in biodegradable polymeric microstructures, a sustained and controlled release of drugs can be achieved [3]. For delivering such encapsulated drugs in the human body, many methods have been suggested. They include the utilization of external magnetic/electric/acoustic fields, bio-actuators, and chemical reactions [4]. Among them, bio-actuators are receiving much attention due to the characteristics of low toxicity and low or no power requirement [5-7]. In fact, bacteria which have self-powered flagella motion have also been proposed for use as multiple spin actuators in microrobots to provide actuation forces [8,9]. The flagellated bacteria, Escherichia coli (E. coli), Salmonella typhimurium (S. typhimurium) and Serratia marcescens (S. marcescens), can be made non-toxic and have been investigated for use in the cancer therapy [10-13].

Our collaborators have fabricated bacteria-based spherical-shaped microstructures for localized drug delivery [14]. Salmonella typhimurium is attached to poly(ethylene glycol) (PEG) by covalently coupling biotin and streptavidin, and the in vitro and in vivo results show that the bacteria-based microrobots move towards tumor sites without additional outside power [14,15].

The motive forces in bacteria-based actuators can be generated by several variations of stimuli-induced movements (or taxes), including aerotaxis (taxis to oxygen), chemotaxis (taxis to a carbon source), magnetotaxis (taxis to magnetic field), and phototaxis (taxis to light) [16-18]. In particular, magneto-aerotatic bacteria have a potential for targeting the cancer cells due to the controllable movements with an external magnetic field [19]. A sperm-driven micro-robot is also possible for developing a new type of DDS [20]. For example, a sperm-tube hybrid system, that also incorporates magnetic nanoparticles, allows controlling motion using an outside field [21].

Selectively attaching bacteria to specific surfaces to increase the directionality of locomotion can shorten the travel time to reach target tumors [22]. There have been several investigations of selective bacteria attachment methods to achieve this. Park et al. attached $S$. marcescens to $30-\mu \mathrm{m}$ sized microcubes made of SU-8 photoresist and demonstrated the directionality of motion [23]. For the preferential attachment of bacteria, bovine serum albumin (BSA), which makes coated surfaces anti-adherent to bacteria, was selectively coated to the microstructures. The measured motilities of cubes were $2.24 \mu \mathrm{m} / \mathrm{s}$ for the control case and $4.72 \mu \mathrm{m} / \mathrm{s}$ for the BSA coated case, respectively.

Another preferential attachment method for bacteria has also been demonstrated, using S. typhimurium and 10- $\mu \mathrm{m}$ sized spherical structures made of PEG with partially coated poly-L-lysine (PLL) [24]. Salmonella typhimurium attaches only to certain restricted regions of PLL. The measured motilities of spherical structures were $0.03 \mu \mathrm{m} / \mathrm{s}$ for the control case and $0.37 \mu \mathrm{m} / \mathrm{s}$ for the PEG spheres partially coated with PLL, respectively. 
The other selective bacterial adhesion method is modifying the surface morphology of structures. Our previous work demonstrated that chemically or physically-modified surfaces can control the bacterial attachments [25-27]. Especially, the dioxygen $\left(\mathrm{O}_{2}\right)$ plasma treatment and X-ray synchrotron irradiation methods modify the surface porosity of structures. Cubic structures of $50-\mu \mathrm{m}$ sides are fabricated using poly-capro-lactone (PCL), which is a biocompatible and biodegradable polymer widely used for drug carrier. The measured motility of $S$. marcescens-attached PCL cubes was $3.89 \mu \mathrm{m} / \mathrm{s}$ for the plasma treatment case [28].

However, these investigations discussed in the above use differently-shaped structures and different bacteria. Thus, the motilities of different investigations are not directly comparable. This paper provides a quantitative evaluation of different selective adhesion methods for the purpose of bacterial motility control. In particular, this paper investigates the methods of BSA coating, X-ray synchrotron irradiation and plasma treatment for preferential attachments of $S$. marcescens and evaluates the motilities of the three different methods. To precisely fabricate cubic microstructures made of PCL, this paper uses the X-ray-lithography-based microfabrication method.

\section{Preparation of Microfabricated Structures and Bacteria-Tethering}

\subsection{Biodegradable Polymeric Microstructures}

For the fabrication of the PCL cubic microstructures, the X-ray-lithography-based microfabrication method is used for accurate dimensional control [29]. First, a PCL film which has a thickness of $50 \mu \mathrm{m}$ is prepared by a spin coating process and a lamination process [30]. Next, X-ray synchrotron irradiation is performed to pattern $50 \mu \mathrm{m} \times 50 \mu \mathrm{m}$ squares. The exposed dose to the PCL microstructures is $10 \mathrm{~J} / \mathrm{cm}^{3}$. For X-ray synchrotron irradiation, the Pohang Accelerator Laboratory (PAL) facility in Korea is used [31]. A $45 \%(w / v)$ potassium hydroxide $(\mathrm{KOH})$ solution in room temperature is used as the developer to separate the microstructures from the substrate and irradiated separation area. After washing with deionized water, the $50-\mu \mathrm{m}$ sized cubic microstructures made of biodegradable PCL polymer are fabricated, as shown in Figure 1.

Figure 1. SEM image of $50-\mu \mathrm{m}$ sized cubic microstructures made of biodegradable poly-capro-lactone (PCL) polymer.

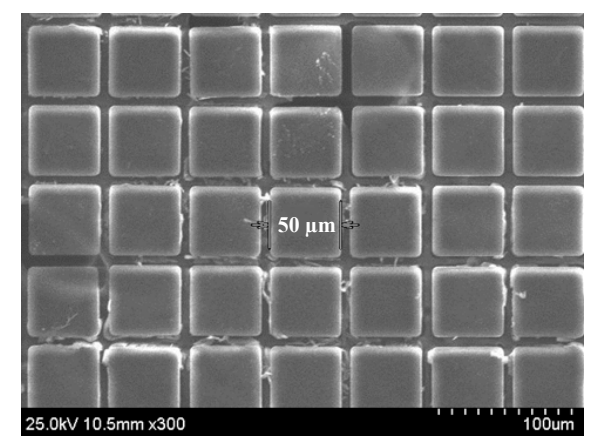

\subsection{Preparation of Treated Microstructures}

For evaluating motility, microstructures are prepared with three different bacterial attachment methods. Firstly, cubic microstructures with five-sides coated with BSA are prepared. The $50-\mu \mathrm{m}$ sized 
cubic microstructures made of PCL polymer are prepared on the substrate. A BSA 5\% solution is poured into the substrate and left for $24 \mathrm{~h}$. After the substrate is dried, the microstructures are extracted from the substrate. Therefore, the microstructures have five BSA-coated sides and one-uncoated side. Secondly, microstructures are prepared by treating the top side with $\mathrm{O}_{2}$ plasma. Using Laboratory Corona Treater (Electro-Technic Products Inc., Chicago, IL USA), only the top side is treated with $\mathrm{O}_{2}$ plasma for $120 \mathrm{~s}$. The original surface of the untreated microstructure is shown in Figure 2a, and the $\mathrm{O}_{2}$ plasma roughened surface is shown in Figure 2b. Thirdly, the microstructures with different surface morphologies using X-ray synchrotron irradiation are also fabricated. After the initial fabrication of cubic microstructures, an additional X-ray irradiation step is performed. The total exposed dose is $2400 \mathrm{~J} / \mathrm{cm}^{3}$. The roughened surface by this method is shown in Figure 2c.

Figure 2. SEM image of surfaces in three different microstructures. (a) Untreated surface of control microstructure. (b) Roughened surface treated by $\mathrm{O}_{2}$ plasma treatment. (c) Roughened surface treated by X-ray irradiation of $2400 \mathrm{~J} / \mathrm{cm}^{3}$.
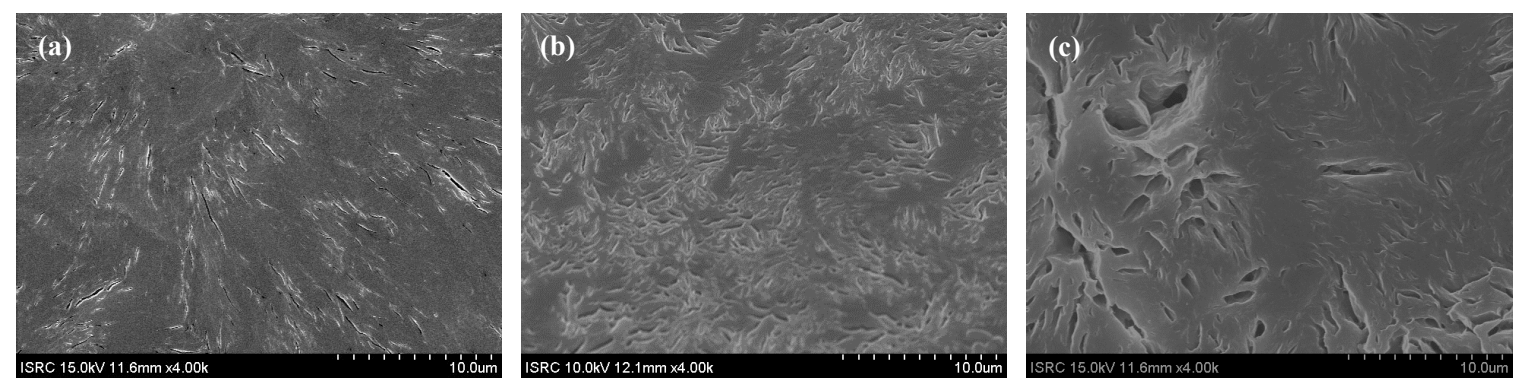

\subsection{Bacteria Culture and Tethering}

Serratia marcescens is selected in this experiment due to their excellent swimming behavior [12]. Serratia marcescens is grown in nutrient agar plate (0.3\% Bacto Beef Extract, $0.5 \%$ Bacto Peptone, and $1.5 \%$ Bacto Agar) for $12 \mathrm{~h}$. Serratia marcescens is cultivated in the shaking incubator $\left(37^{\circ} \mathrm{C}, 100 \mathrm{rpm}\right)$ for $12 \mathrm{~h}$ with liquid media contained nutrient broth $(0.3 \%$ Bacto Beef Extract and $0.5 \%$ Bacto Peptone, $500 \mathrm{~mL}$ deionized water). The bacterial density of the final harvested bacteria solution is measured using an UV spectrophotometer at a wavelength of $600 \mathrm{~nm}$ (UV/VIS Spectrophotometer-Optizen $2120 \mathrm{UV}$, Mechasys, Anyang, Korea). At an optical density of $1\left(\mathrm{OD}_{600}=1\right)$, S. marcescens is used for bacteria tethering.

The solution containing $S$. marcescens is poured to the prepared microstructures. For the control case, bacteria attach to random surfaces. In the BSA coating method, bacteria attach only to the BSA-uncoated side. In the X-ray irradiation methods, the X-ray irradiation treatment seems to roughen all surfaces, and generally more bacteria attach to all surfaces, when compared to the untreated control microstructure. This is because X-ray synchrotron irradiation has a high penetration property, and that makes modifying only the top side difficult. In the $\mathrm{O}_{2}$ plasma treatment case, only the top side of the microstructure is significantly roughened, and more bacteria attach to only the top side. The schematic diagrams of bacteria tethering are shown in Figure 3.

All experiments are performed three times on separate days. In each experiment, one hundred microstructures are investigated. The number of bacteria tethering is similar for each surface treatment method. Therefore, representative microstructures are chosen for showing the density of bacterial attachments. 
Figure 4 shows the results of bacterial attachments on PCL microstructure surfaces. In case of the untreated control microstructure, the number of attached bacteria to the area of $600 \mu \mathrm{m}^{2}$ is 20 , as shown in Figure 4a. The BSA coating method, which retards bacterial attachments, shows that the number of attached bacteria to the same area is 9, as shown in Figure $4 \mathrm{~b}$. The number of attached bacteria to the same area for the X-ray irradiation treatment in Figure $4 \mathrm{c}$ is 35 , and that for the plasma treatment in Figure $4 \mathrm{~d}$ is 45 , respectively. Because the methods of X-ray irradiation and plasma treatment roughen PCL surfaces differently, the number of tethered bacteria is different.

Figure 3. Schematic diagrams of bacteria-tethering. (a) Untreated control microstructure. (b) Microstructure coated on five-sides by bovine serum albumin (BSA). (c) X-ray irradiation treated microstructure. (d) Microstructure treated on one side by $\mathrm{O}_{2}$ plasma.
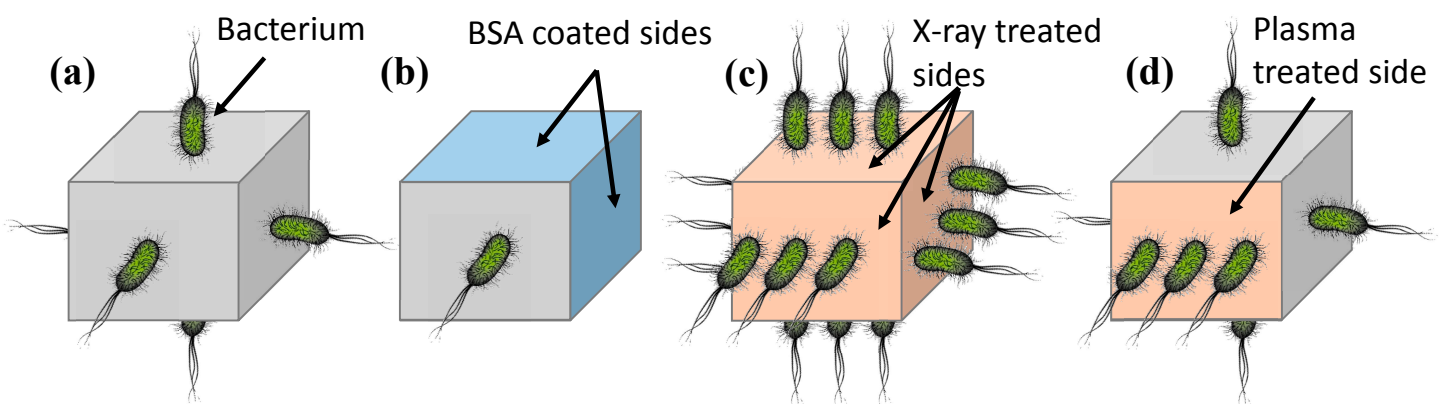

Figure 4. SEM images of bacterial attachment on PCL microstructure surfaces. (a) Bacteria-attached polymeric surface of untreated control microstructure. The number of bacteria attached to the area of $600 \mu \mathrm{m}^{2}$ is 20 . (b) Bacteria-attached polymeric surface with BSA treatment. The number of bacteria attached to the area of $600 \mu \mathrm{m}^{2}$ is 9 . (c) Bacteria-attached polymeric surface with X-ray irradiation of $2400 \mathrm{~J} / \mathrm{cm}^{3}$. The number of bacteria attached to the area of $600 \mu \mathrm{m}^{2}$ is 35 . (d) Bacteria-attached polymeric surface with plasma treatment. The number of bacteria attached to the area of $600 \mu \mathrm{m}^{2}$ is 45 .
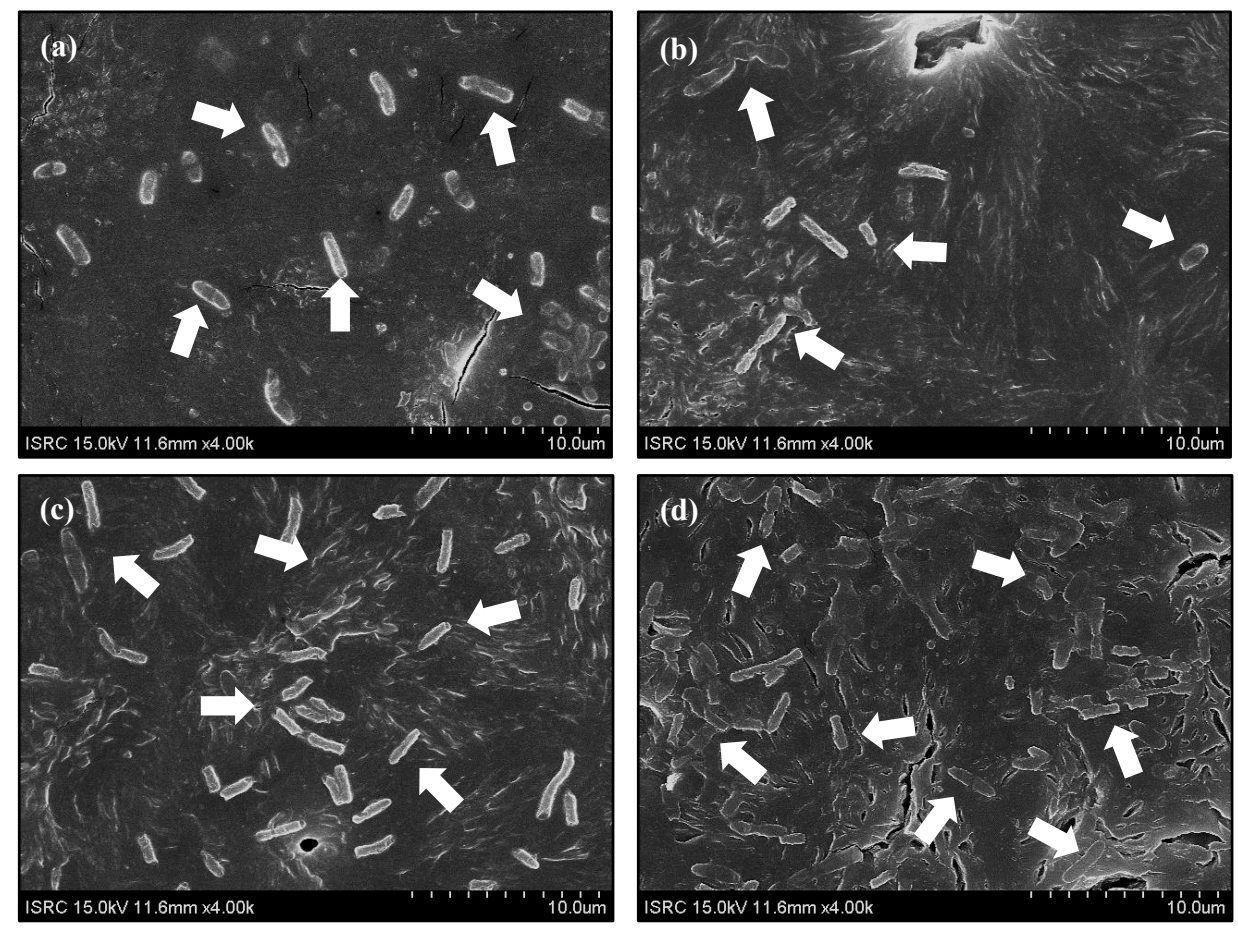


\section{Motility Measurements}

Motility measurements of the above-mentioned four types of bacteria-tethered microactuators are performed in a 4-inch petri dish without a channel. In the petri dish, $10 \mathrm{~mL}$ of a motility medium $(0.1 \mathrm{M}$ potassium phosphate tribasic, $10^{-4} \mathrm{M}$ ethylenediaminetetraacetic acid (EDTA), $0.067 \mathrm{M}$ sodium chloride, $0.01 \mathrm{M}$ glucose, and $0.002 \%$ Tween-20, $\mathrm{pH} 7.0$ ) is poured, and the motilities of bacteria-tethered microactuators are observed. A set of continuous frame images of bacteria-tethered microactuators are captured by an inverted microscope (IX 51, Olympus, Tokyo, Japan). The actual moving distances between the continuous frame images during $1 \mathrm{~min}$ are calculated based on the pixel to pixel distances, using the centroid coordinates of the microstructures. Since the centroid pixel values of the microstructures and the actual length per pixel are known, the difference of the centroid coordinates between the start and the end positions is used to calculate the linear distances and average velocities. Note that all microstructures show some rotational movements. Bacteria adhere to sides at random sites and random directions, which in turn leads to some random motion.

As shown in Figure 5a, the average velocity of the untreated control microstructure is $0.31 \mu \mathrm{m} / \mathrm{s}$ with a rotational movement. Because of the random attachments, the control case shows motility without a preferential direction. In contrast, the three developed bacteria-tethered microactuators show defined directional motility. With the BSA coating method, the average velocity of microstructure is $1.40 \mu \mathrm{m} / \mathrm{s}$, as shown in Figure 5b. The average velocities of the microstructures with the X-ray irradiation treatment and the plasma treatment are $0.82 \mu \mathrm{m} / \mathrm{s}$ and $3.89 \mu \mathrm{m} / \mathrm{s}$, respectively, as shown in Figure $5 \mathrm{c}$,d. The results are summarized in Table 1 and Figure 6.

The microactuator with roughened surfaces treated by X-ray irradiation has 2.64 times higher velocity than the control case. This difference comes from the number of bacterial attachments to surfaces. The velocity of microactuator with one side treated by $\mathrm{O}_{2}$ plasma shows 12.54 times higher than that of the untreated control case and 2.78 times higher than that of the BSA coating case. Therefore, plasma treatment is a better method for enhancing the directionality of motion, when compared to the BSA coating method or the X-ray irradiation method. Note that if a microstructure is fabricated with one-side treated by plasma and the other sides treated by BSA coating, the microactuator will show a faster velocity with further improved directionality.

Figure 5. Images of individual video frames of movement of bacteria-tethered cubic microstructures captured by microscope. (a) Untreated control microstructure. (b) Microstructure coated on five-sides by BSA. (c) X-ray irradiation treated microstructure. (d) Microstructure treated on one side by plasma.

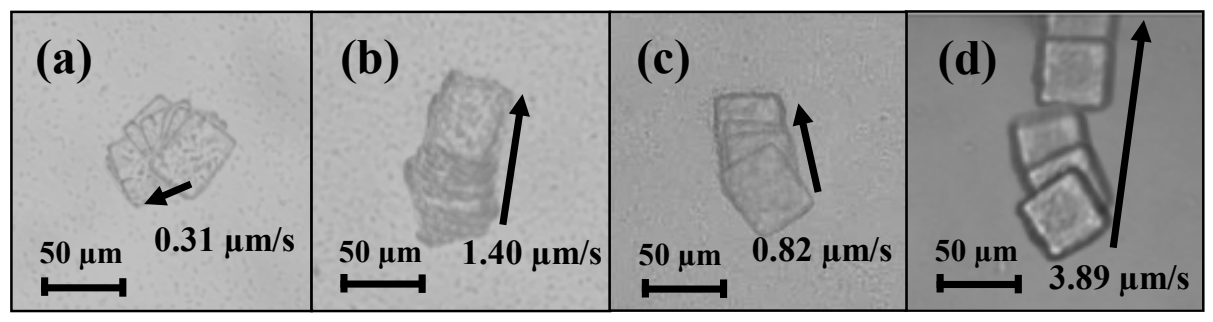


Table 1. Directional moving distances and average velocities of microactuators.

\begin{tabular}{ccc}
\hline Preferential tethering method & Total directional moving distance during $\mathbf{1} \mathbf{~} \mathbf{m i n}(\boldsymbol{\mu m})$ & Average velocity $(\boldsymbol{\mu m} / \mathbf{s})$ \\
\hline Untreated & 18.6 & 0.31 \\
BSA coating & 84 & 1.40 \\
X-ray irradiation treatment & 49.2 & 0.82 \\
Plasma treatment & 233.4 & 3.89 \\
\hline
\end{tabular}

Figure 6. Average velocities of microactuators.

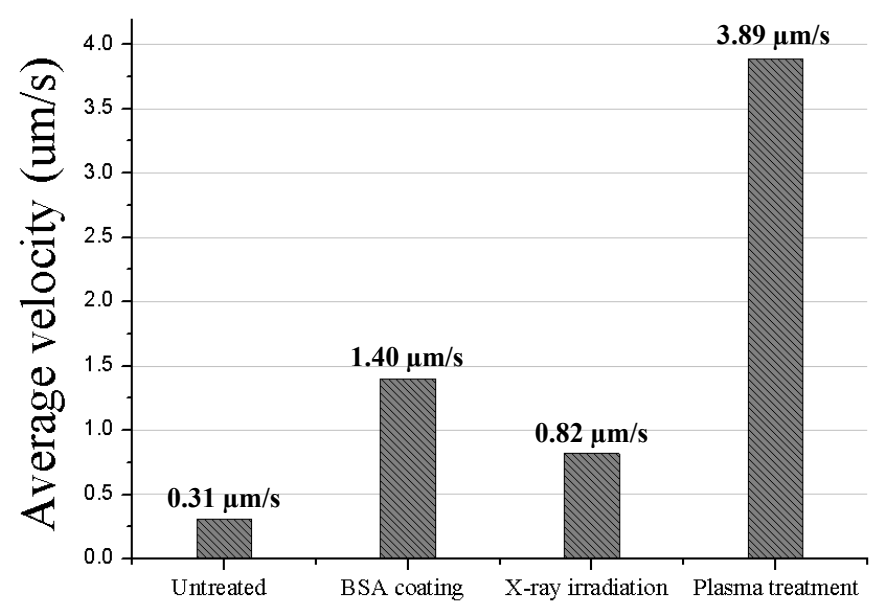

\section{Conclusions}

This paper investigated three selective adhesion methods for attaching flagellated bacteria on the preferred surfaces of cubic polymeric microactuators to improve the directionality of actuation. Serratia marcescens and PCL microstructures were used in this paper. The preferential tethering of bacteria was performed using the methods of BSA coating, X-ray irradiation and plasma treatment. Among these methods, the plasma treatment showed the most desirable characteristics. We are investigating further uses of the developed plasma-treated, biodegradable polymeric microactuators with attenuated bacteria as actuators for delivering drugs to tumor sites. It should be noted that this is a long-term project which requires substantially more research.

\section{Acknowledgments}

We thank Mr. Jong Hyun Park for the experimental assistance. This research was supported by Future-Based Technology Development Program (Nano Fields) through the National Research Foundation of Korea (NRF) funded by the Ministry of Science, ICT and Future Planning (2009-0082952). The X-ray irradiation process was additionally supported by the Pohang Accelerator Laboratory (PAL; http://pal.postech.ac.kr/).

\section{Author Contributions}

Hyung Jung Yoo fabricated the devices and performed the experiments. Sangmin Lee designed the initial experiments. Dong-il Cho supervised the entire work and is the corresponding author. 


\section{Supplementary Materials}

Supplementary materials can be accessed at: http://www.mdpi.com/2072-666X/5/4/1287/s1.

\section{Conflicts of Interest}

The authors declare no conflict of interest.

\section{References}

1. Allen, T.M.; Cullis, P.R. Drug delivery systems: entering the mainstream. Science 2004, 303, $1818-1822$.

2. Zafar Razzacki, S.; Thwar, P.K.; Yang, M.; Ugaz, V.M.; Burns, M.A. Integrated microsystems for controlled drug delivery. Adv. Drug Delivery Rev. 2004, 56, 185-198.

3. Jain, R.; Shah, N.H.; Malick, A.W.; Rhodes, C.T. Controlled drug delivery by biodegradable poly (ester) devices: Different preparative approaches. Drug Dev. Ind. Pharm. 1998, 24, 703-727.

4. Feng, J.; Cho, S.K. Mini and micro propulsion for medical swimmers. Micromachines 2014, 5, 97-113.

5. Bogue, R. The development of medical microrobots: A review of progress. Ind. Robot. 2008, 35, 294-299.

6. Sowa, Y.; Berry, R.M. Bacterial flagellar motor. Q. Rev. Biophys. 2008, 41, 103-132.

7. Minamino, T.; Imada, K.; Namba, K. Molecular motors of the bacterial flagella. Curr. Opin. Struc. Biol. 2008, 18, 693-701.

8. Copeland, M.F.; Weibel, D.B. Bacterial swarming: A model system for studying dynamic self-assembly. Soft Matter 2009, 5, 1174-1187.

9. Berg, H.C.; Robert A.A. Bacteria swim by rotating their flagellar filaments. Nature 1973, 380-382.

10. Forbes, N.S. Engineering the perfect (bacterial) cancer therapy. Nat. Rev. Cancer 2010, 10, 785-794.

11. Darnton, N.; Turner, L.; Breuer, K.; Berg, H.C. Moving fluid with bacterial carpets. Biophys. J. 2004, 86, 1863-1870.

12. Le, U.N.; Kim, H.S.; Kwon, J.S.; Kim, M.Y.; Nguyen, V.H.; Jiang, S.N.; Lee, B.I.; Hong, Y.; Shin, M.G.; Rhee, J.H.; Bom, H.S.; Ahn, Y.; Gambhir, S.S.; Choy, H.E.; Min, J.J. Engineering and visualization of bacteria for targeting infarcted myocardium. Mol. Ther. 2011, 19, 951-959.

13. Patyar, S.; Joshi, R.; Byrav, D.P.; Prakash, A.; Medhi, B.; Das, B.K. Bacteria in cancer therapy: A novel experimental strategy. J. Biomed. Sci. 2010, 17, 21-30.

14. Park, S.J.; Park, S.H.; Cho, S.; Kim, D.M.; Lee, Y.; Ko, S.Y.; Hong, Y.; Choy, H.E.; Min, J.J.; Park, J.O.; Park, S. New paradigm for tumor theranostic methodology using bacteria-based microrobot. Sci. Rep. 2013, 3, 3394.

15. Taherkhani, S.; Mohammadi, M.; Daoud, J.; Martel, S.; Tabrizian, M. Covalent binding of nanoliposomes to the surface of magnetotactic bacteria for the synthesis of self-propelled therapeutic agents. ACS Nano 2014, 8, 5049-5060.

16. Kim, D.; Liu, A.; Diller, E.; Sitti, M. Chemotactic steering of bacteria propelled microbeads. Biomed. Microdevices 2012, 14, 1009-1017.

17. Taylor, B.L.; Zhulin, I.B.; Johnson, M.S. Aerotaxis and other energy-sensing behavior in bacteria. Annu. Rev. Microbiol. 1999, 53, 103-128. 
18. Steager, E.B.; Sakar, M.S.; Kim, D.H.; Kumar, V.; Pappas, G.J.; Kim, M.J. Electrokinetic and optical control of bacterial microrobots. J. Micromech. Microeng. 2011, 21, 035001.

19. Frankel, R.B.; Bazylinski, D.A.; Johnson, M.S.; Taylor, B.L. Magneto-aerotaxis in marine coccoid bacteria. Biophys. J. 1997, 73, 994-1000.

20. Magdanz, V.; Sanchez, S.; Schmidt, O.G. Development of a sperm-flagella driven micro-bio-robot. Adv. Mater. 2013, 25, 6581-6588.

21. Khalil, I.S.; Magdanz, V.; Sanchez, S.; Schmidt, O.G.; Misra, S. Biocompatible, accurate, and fully autonomous: A sperm-driven micro-bio-robot. J. Micro Bio Robot. 2014, 9, 79-86.

22. Dmochowski, R.R.; Staskin, D.R. Advances in drug delivery: Improved bioavailability and drug effect. Curr. Urol. Rep. 2002, 3, 439-444.

23. Park, S.J.; Bae, H.; Kim, J.; Lim, B.; Park, J.O.; Park, S. Motility enhancement of bacteria actuated microstructures using selective bacteria adhesion. Lab Chip 2010, 10, 1706-1711.

24. Cho, S.; Park, S.J.; Ko, S.Y.; Park, J.O.; Park, S. Development of bacteria-based microrobot using biocompatible poly(ethylene glycol). Biomed. Microdevices 2012, 14, 1019-1025.

25. Choi, H.M.; Koo, K.I.; Park, S.G.; Jeong, M.J.; Kim, G.S.; Park, J.H.; Lim, J.M.; Chung, W.J.; Lee, S.H.; Jin, S.W.; Lee, Y.S.; Park, T.H.; Yoo, J.Y.; Cho, D.I. Improvement of bacterial tethering using both physical and chemical surface modification for flagella spin actuators. Sens. Actuators B Chem. 2007, 123, 269-276.

26. Lee, S.; Hong, S.J.; Yoo, H.J.; Cho, D.I. Surface energy modification of biodegradable polymeric surfaces. In Proceedings of the International Symposium on Microchemistry and Microsystems 2011 in Conjunction with the Korean BioChip Society Spring Meeting, Seoul, Korea, 2-4 June 2011.

27. Yoo, H.J.; Lee, S.M.; Ahn, J.H.; Oh, S.J.; Song, B.H.; Kim, S.J.; Seo, J.M.; Kim, T.Y.; Cho, D.I. Surface energy modification method using X-ray synchrotron irradiation for controlling bacterial adhesion on biodegradable-polymer structures for bacteria-flagellated microrobots. In Proceedings of the 2013 International Conference on Control, Automation and Systems, Gwangju, Korea, 20-23 October 2013.

28. Yoo, H.J.; Park, J.H.; Lee, S.M.; Ahn, J.H.; Oh, S.J.; Song, B.H.; Shin, J.Y.; Cho, D.I. Motility of bacteria-attached microstructures: Bovine serum albumin coating and plasma treatment cases. In Proceedings of the 7th Asia-Pacific Conference of Transducers and Micro-Nano Technology, Daegu, Korea, 29 June-2 July 2014.

29. Cho, D.I.; Yoo, H.J.; Lee, S.; Hong, S.J.; Seo, J.M.; Kim, T.Y.; Kim, S.J. Trends in drug-delivery-system microfabrication and a new scalable technology using X-ray synchrotron irradiation. In Proceedings of the 17th International Conference on Solid-State Sensors, Actuators and Microsystems, Barcelona, Spain, 16-20 June 2013.

30. Yoo, H.J.; Lee, S.M.; Ahn, J.H.; Cho, D.I. Drug-loaded cubic micro-chamber made of a biodegradable polymer for bacteria-based drug delivery. In Proceedings of the 8th IEEE International Conference on Nano/Micro Engineered and Molecular Systems, Suzhou, China, 7-10 April 2013.

31. Pohang Accelerator laboratory. Available online: http://pal.postech.ac.kr (accessed on 24 November 2014).

(C) 2014 by the authors; licensee MDPI, Basel, Switzerland. This article is an open access article distributed under the terms and conditions of the Creative Commons Attribution license (http://creativecommons.org/licenses/by/4.0/). 